\title{
Eszter Pabis: Literarische Grenzgänge. Dimensionen der Fremdheit in der deutschsprachigen Gegenwartsliteratur der Schweiz
}

Wien: Praesens 2017 - ISBN 978-3-7069-0930-3 - 28,00€

Im abschließenden Kapitel ihrer ersten Monografie, Die Schweiz als Erzählung. Nationale und narrative Identitätskonstruktionen in Max Frischs Stiller, Wilhelm Tell für die Schule und Dienstbüchlein (2010), bespricht Eszter Pabis diverse theoretische Ansätze und Konzepte (u.a. eine »Ästhetik der Differenz« und die literaturwissenschaftliche Xenologie), die von der strukturellen Analogie ästhetischer und kultureller Erfahrungen ausgehend den Beitrag der Literatur zur Konstruktion von Alterität und Zugehörigkeit fokussieren. In dem vorliegenden Buch, $\mathrm{Li}$ terarische Grenzgänge. Dimensionen der Fremdheit in der deutschsprachigen Gegenwartsliteratur der Schweiz, berücksichtigt Pabis ebenfalls die Interdependenz zwischen ästhetischen Verfahren und der transkulturellen Konstellation sowie die Thematik der Grenzgängerschaft und die besondere Sprachlichkeit des grenzüberschreitenden Schreibens. Dadurch leistet der Band einen Beitrag zur Diskussion um Zusammenhänge zwischen sprachlichästhetischer und migrationsbedingter Verfremdung und Polyphonie, welche eine neue Kontextualisierung des Schaffens zeitgenössischer und klassischer Autorinnen und Autoren ermöglicht.

Die zehn Beiträge des Bandes größtenteils narratologische Untersuchungen zu Prosatexten jüngerer Autoren und Autorinnen aus der Deutsch- schweiz - sind vielfältig, aber systematisch ausgerichtet. Die einführenden Theoriekapitel problematisieren neuere Beschreibungs- und Interpretationsmodelle der transnationalen, postkolonialen oder polyphonen Schweizer Literatur und jene Grundbegriffe (vor allem aus der Xenologie), die für die Textanalysen erkenntnisleitend sind, so u.a. Fremdverstehen, Verfremdung, Grenzüberschreitung oder Dialogizität. Die anschließenden Textanalysen behandeln unterschiedliche literarische Spielarten der Fremdheit und widmen sich je einem repräsentativen Werk - mit Ausnahme der Kapitel drei und sechs, die mehrere Grenzgängergeschichten bzw. beinahe das ganze Euvre Max Frischs überblicken. Als Fazit werden in der Arbeit auch weiterführende Forschungspotentiale aufgezeigt. Zum analysierten Textkorpus gehören neben vielrezipierten Texten von bereits kanonisierten Autoren (wie Max Frisch, Hermann Burger, Urs Widmer oder Markus Werner) auch Erzähltexte jüngerer bzw. eingewanderter Autorinnen und Autoren (Martin R. Dean, Angelika Overath oder Melinda Nadj-Abonji). Es lässt sich zwar bezweifeln, ob die Auswahl repräsentativ ist, in der Zusammenstellung der Beiträge spiegelt sich aber auf jeden Fall die Diversität und Komplexität des Gegenstandes. Im Folgenden werden die Einzeltextstudien nicht der Reihenfolge oder 
der Relevanz nach kurz vorgestellt, sondern von zentralen Grundbegriffen und Problemfeldern ausgehend, welche narratologische Zusammenhänge zwischen kulturellen und ästhetischen Alteritätsphänomenen und Fremdheitserfahrungen betreffen und eine Engführung textnaher Interpretation und theoretischer Fragestellungen ermöglichen.

Erstens erweisen sich die Begriffe der Grenze und Grenzüberschreitung für Pabis als aufschlussreich, um die Problematik der Fremde und Verfremdung als paradigmatische Merkmale literarischer Texte mit weiteren Alteritätsphänomenen $\mathrm{zu}$ verbinden und Einsichten aus der Xenologie in der literarischen Textanalyse produktiv einzusetzen. In Anlehnung an bekannte Thesen von Bhabha, Waldenfels, Foucault und Iser beschreibt Pabis Grenzräume als grundsätzlich ambivalent, dialektisch, hybrid und transitorisch: Sie machen die diskursive Konstruiertheit und die Kontingenz der Grenzziehungen generell erkennbar. Grenzen verfügen, so Pabis, auch über eine anthropologische Dimension und sie gehören zu den Grundkategorien der Literatur(-wissenschaft) und Ästhetik. Die narratologischen Strukturen, die Sprache und Metaphorik der Texte Nadj-Abonjis (Tauben fliegen auf, 2010) und Ilma Rakusas (Mehr Meer, 2009) modellieren beispielsweise die permanente Verschiebung und Vermischung von Identitätskonstruktionen und Bedeutungen und inszenieren außerdem nicht nur die Grenzüberschreitung als räumliche oder politische Erfahrung, sondern auch Übergangserlebnisse, Grenzzonen und Schwellenerfahrungen wie Sexualität, Tod oder Krankheit.
Angelika Overaths Flughafenfische (2009) ist zwar weder ein Migrationsroman noch ein Reiseroman, aber auf jeden Fall ein Transit-Narrativ, dessen einziger Handlungsschauplatz ein Flughafen ist, d.h. ein Schwellenort oder ein exemplarischer Nichtort im Sinne Augés. Pabis gewährt einen Einblick in die paradoxen Strukturen des palimpsestähnlich überlagerten Nichtortes (so u.a. in die Dynamik der Fortbewegung bzw. Deplatziertheit und der Statik bzw. der Uniformisierung im Verkehrs- und Konsumraum) und verbindet diese mit dem Textraum des Romans, der sich durch komplizierte intra- und intertextuelle Verweisstrukturen auszeichnet und letztendlich auch das komplexe Verhältnis zwischen Text, Raum und Wahrnehmung thematisiert. Pabis kommt in der textnahen Analyse zu dem Schluss, dass der Roman auf räumlichen Ordnungen und Strukturen aufbaut und sich dem beschriebenen Aquarium analog verhält: Flughafenfische liest sich damit als ein Text über den Raum.

Die Deutungsansätze des Postkolonialismus bezieht Pabis auf das latente Weiterwirken hegemonialer Diskurse und das Fortleben ethnozentrischer Einstellungen und asymmetrischer Machtverhältnisse. Urs Widmers Im Kongo (1996) wird als Gesellschaftssatire und Erinnerungsroman interpretiert, in dem koloniale Gewaltexzesse, die Schweizer Kriegsvergangenheit und Alltagsrassismen in der Migrationsgesellschaft in einer magisch-realistischen Schreibweise aufeinander bezogen werden. Die Verknüpfungen kolonialer und postkolonialer Gewalterfahrungen, die Auswirkungen his- 
torischer Traumata, ihre Medialität und Erzählweisen werden in Martin R. Deans Roman Meine Väter (2003) ähnlich thematisiert und narratologisch modelliert. Das »Vaterbuch« problematisiert die (u.a. intermedialen) Zusammenhänge zwischen struktureller, symbolischer oder konkreter Gewalt, ästhetischer Tätigkeit und geschichtlicher Spurensuche und illustriert damit das nachkoloniale Fortbestehen von sexuellen oder patriarchalen $\mathrm{Ge}$ waltstrukturen.

Die Fremdheit bzw. die innersprachliche Polyphonie von sprachlich hybriden Textstellen ist zentral für die meisten Textanalysen, insbesondere für die Interpretation der Perspektiven- und Stimmenvielfalt in Beat Sterchis Roman Blösch (1983), in dem kulturelle Grenzerfahrungen (Geburt, Leben, Tod) und der chiastische Charakter der Selbst- und Fremderfahrung in einem besonderen Kontext behandelt werden: Die Analogie von Mensch und Tier manifestiert sich in der Sprachlosigkeit der Figur des Fremden und prägt den narrativen Diskurs als Parallele zwischen Menschen- und Tierkörpern und dem Sprachkörper des Romans: Demontage und Subversion bestimmen sowohl im konkreten Sinn die Handlung und die Thematik (der Schauplatz ist ein Schlachthof, die Protagonisten sind eine Kuh und ein Gastarbeiter) als auch die Sprache und Narrativik.

Die unauflösbare Fremdheit des Fremdwortes entlarvt in Hermann Burgers Brieferzählung Diabelli, Prestidigitateur (1979) die Kontingenz, die Selbstreferenzialität, die Künstlichkeit und Simulierbarkeit von Sprache und Identität. Die konstitutive Rolle der radikalen sprachlichen Fremd- heit interpretiert Pabis im Kontext der Ambivalenz von Auflösung und Vervielfältigung des Subjekts, welche thematische Leitmotive des Textes sind (Diabellis Zauberkunst zielt auf Selbstauflösung und Selbstvervielfältigung) und auch die Position und Konstituierung des Erzählers und des (postmodernen) Subjektes kennzeichnen. An diesem Punkt zeigt sich nicht nur die Relevanz der Analysekategorien der Redevielfalt, der Mehr- und Fremdsprachigkeit und der sprachlichen Hybridisierung für die Interpretation literarischer Texte und kultureller Identitätskonstruktionen, sondern auch, welche weiterführenden Perspektiven die vorgelegte Arbeit eröffnet. Die Untersuchung ließe sich nämlich produktiv auf Texte erweitern, die die mediale Diglossie literarisch reflektieren, d.h. mit einer Differenz zwischen dem Eigenen und dem Fremden im Spannungsfeld von Mundart und Schriftsprache operieren.

Das Kapitel über die Dimensionen der Fremdheit in Max Frischs Werk stützt sich größtenteils auf die Interpretationen in der Schweiz als Erzählung (2010), diese werden aber ergänzt bzw. aus einer neuen Perspektive betrachtet durch eine Fokussierung auf den Zusammenhang zwischen Befremdung und Bildnisverbot, Fremderfahrung und Identitätskonstruktion durch Befreiung aus festgelegten Rollen. Die Analyse des Stiller (1954) behandelt die Interdependenz von persönlicher und nationaler Identität sowie den Zusammenhang zwischen Reise- und Fremdheitserfahrungen und Identitätskonstruktion. In Homo faber (1957) stehen ebenfalls Transitsituationen im Mittelpunkt bzw. die innersprachliche Polyphonie des Ro- 
mans, die eine Interdependenz von Bildnis und Sprache, von ästhetischen und kulturellen Fremdheitserfahrungen vermittelt. Auch das Drama Andorra (1961) zeigt den Zusammenhang zwischen Sprache, Bildnis und Identität sowie die fatalen Konsequenzen der antisemitischen Bildnismacherei auf. In der Umdichtung des Schweizer Gründungsmythos in Wilhelm Tell für die Schule (1970) verschränken sich die Fremdheitserfahrungen des Protagonisten mit der parodistisch verfremdeten Rhetorik nationaler Identitätsbildung: Die Relevanz der Intertexte und die rhetorischen Verfahren der Satire gehören aber auch zu jenen Aspekten der Fremdheit, welche die Wechselwirkung ästhetischer und kultureller Alteritätsphänomene illustrieren.

Alle im Band besprochenen Texte verfügen auch über eine gedächtnistheoretische Relevanz. Der ägyptische Heinrich und Im Kongo leisten einen Beitrag zur Diskussion um die Schweizer Vergangenheitsbewältigung und die Migrationsromane, die ähnlich wie Nadj-Abonjis interkultureller Familienroman Tauben fliegen auf grenzüberschreitende
Bewegungen zwischen Ostmitteleuropa und dem Westen beschreiben, gewähren einen Einblick in die Verschränkungen von Erinnerungskultur, Geschichtspolitik und kulturellem Gedächtnis in westlichen und in postkommunistischen Gesellschaften. In diesem Kontext ließen sich weitere Grenzgängergeschichten (z.B. von deutsch-ungarischen Autorinnen) auf Verflechtungen zwischen Schreibstrategien und migratorischen Identitätsmustern hin untersuchen.

Ein Verdienst des Bandes ist es, dass er nebst der Neueinordnung bzw. Neuinterpretation des Schaffens Deutschschweizer Autorinnen und Autoren die Relevanz der Ansätze aus der Xenologie für die Literaturanalyse beleuchtet. Damit erweisen sich die Literarischen Grenzgänge als eine anregende Lektüre nicht nur für Germanisten bzw. Erforscherinnen der Schweizer Literatur und Kultur, sondern auch für alle, die aktuelle Fragen der Erinnerungskultur und Geschichtspolitik in einer transnationalen Perspektive verstehen möchten.

Anett Szegvölgyi-Pócsik

\section{Birgit Huemer, Eve Lejot, Katrien L. B. Deroey (Hg.): Academic writing across languages: multilingual and contrastive approaches in higher education. L'écriture académique à travers les langues: approches multilingues et contrastives dans l'enseignement supérieur. Wissenschaftliches Schreiben sprachübergreifend: mehrsprachige und kontrastive Ansätze in der Hochschulbildung}

Wien: Böhlau Verlag 2019 - ISBN: 978-3-205-20705-4 - 37,99€

In dem Sammelband werden Ansätze zum wissenschaftlichen Schreiben in verschiedenen Sprachen als wichtige Aspekte von Hochschulbildung disku- tiert. Das Buch ist aus dem gleichnamigen Symposium hervorgegangen, das 2016 an der Universität Luxemburg stattfand. Gleichzeitig handelt 